\title{
Error Analysis for Spline Collocation Methods With Application to Knot Selection
}

\author{
By J. Christiansen and R. D. Russell*
}

\begin{abstract}
Some collocation schemes used to solve $m$ th order ordinary differential equations are known to display superconvergence at the mesh points. Here we show that some such schemes have additional superconvergence points for the approximate solution and all of its derivatives. Using such points, we argue that a mesh selection scheme introduced by Dodson can be expected to perform well under general circumstances. A numerical example is given to demonstrate the new superconvergence results.
\end{abstract}

I. Introduction. The authors recently considered [4] the problem of adaptive mesh selection when solving boundary value problems by spline collocation. One of the most straightforward and efficient schemes is one presented by de Boor [1]. It was proposed for the collocation methods which display superconvergence at the mesh points (see de Boor and Swartz [2]) so that mesh selection based on local behavior is valid. When using splines of degree $k-1$, the scheme involves approximating the $k$ th derivative of the exact solution from values of the $(k-1)$ st derivative of a spline approximation. There does not seem to have been any proof that such an approximation can converge. A new result from [4] can be used to show that a convergent approximation to the $k$ th derivative is obtainable in this way and that the scheme yields mesh distributions which equalize or equidistribute [5] the dominant terms in the error, provided one starts sufficiently close to the solution.

II. The Structure of the Error. The collocation method is used to approximate the solution $u(x)$ to the problem

$$
\begin{gathered}
L u(x)=u^{(m)}(x)+\sum_{j=0}^{m-1} a_{j}(x) u^{(j)}(x)=f(x) \quad(a \leqslant x \leqslant b), \\
\sum_{j=0}^{m-1}\left(\alpha_{i j} u^{(j)}(a)+\beta_{i j} u^{(j)}(b)\right)=\gamma_{i} \quad(1 \leqslant i \leqslant m) .
\end{gathered}
$$

In particular, suppose the mesh $\pi: a=x_{1}<x_{2}<\cdots<x_{N+1}=b$ is given with $h=$ $\max \left(h_{i}\right)=\max \left(x_{i+1}-x_{i}\right)$ and

$$
\frac{h}{\min \left(h_{i}\right)} \leqslant K
$$

where $K$ is a fixed constant independent of $\pi$. Then a piecewise polynomial $v(x)$ of

Received February 24, 1977; revised July 10, 1977.

AMS (MOS) subject classifications (1970). Primary $65 \mathrm{~L} 10$.

* This work was supported in part under NRC (Canada) Grant A8781.

Copyright $\odot 1978$, American Mathematical Society 
degree $<k$ on each $\left[x_{i}, x_{i+1}\right]$ and in $C^{(m-1)}[a, b]$ is determined by requiring it to satisfy (2.2) and (2.1) at $(k-m)$ points in each $\left[x_{i}, x_{i+1}\right](1 \leqslant i \leqslant N)$. These (collocation) points in $\left[x_{i}, x_{i+1}\right]$ are chosen as

$$
\frac{x_{i}+x_{i+1}}{2}+\rho_{j} \frac{h_{i}}{2} \quad(1 \leqslant j \leqslant k-m)
$$

where $\left\{\rho_{j}: 1 \leqslant j \leqslant k-m\right\}$ consists of either the Gauss or Lobatto points in $[-1,1]$. Although we consider only the linear problem (2.1), (2.2), the results generalize readily to the nonlinear case by making use of results in [2] (see especially Eq. (3.26)).

The form of the collocation error is given by

THEOREM 2.1. Suppose that $a_{j}(x) \in C^{2(k-m)}[a, b] ;(2.1),(2.2)$ has a unique solution $u(x) \in C^{(2 k+1)}[a, b]$; and that (2.1), (2.2) has a Green's function $G(x, t)$ satisfying

$$
\left\|\frac{\partial^{j}}{\partial x^{j}} \frac{\partial^{l}}{\partial t^{l}} G(x, \cdot)\right\| \leqslant C \quad(0 \leqslant j \leqslant m-1,0 \leqslant l \leqslant k-m),
$$

where $G(x, \cdot)$ is an element of $C^{(m)}[a, x] \times C^{(m)}[x, b]$. Then if

$$
d= \begin{cases}2(k-m) & \text { for the Gauss points, } \\ 2(k-m-1) & \text { for the Lobatto points }\end{cases}
$$

and $d>k$, the error for $x \in\left(x_{i}, x_{i+1}\right)$ satisfies

$$
\begin{aligned}
e^{(j)}(x) & \equiv u^{(j)}(x)-v^{(j)}(x) \\
& =u^{(k)}\left(x_{i}\right)\left(\frac{h_{i}}{2}\right)^{k-j} P^{(j)}\left(\frac{2}{h_{i}}\left(x-x_{i+1 / 2}\right)\right)+O\left(h^{k-j+1}\right) \quad(0 \leqslant j \leqslant k),
\end{aligned}
$$

where $x_{i+1 / 2}=x_{i}+h_{i} / 2$ and the polynomial

$$
P(\xi)=\frac{1}{(k-m) !(m-1) !} \int_{-1}^{\xi}(t-\xi)^{m-1} \prod_{j=1}^{k-m}\left(t-\rho_{j}\right) d t .
$$

Proof. Using the hypotheses of the theorem, it was shown in [2, Theorem 4.1] that

$$
\left\|u^{(j)}-v^{(j)}\right\|_{\infty}=O\left(h^{k-j}\right) \quad(0 \leqslant j \leqslant m)
$$

and also that

$$
u^{(j)}\left(x_{i}\right)-v^{(j)}\left(x_{i}\right)=O\left(h^{d}\right) \quad(1 \leqslant i \leqslant N+1 ; 0 \leqslant j \leqslant m-1) .
$$

Extending this analysis somewhat in the appendix of [4], the authors showed that

$$
e(x) \equiv u(x)-v(x)=u^{(k)}\left(x_{i}\right)\left(\frac{h_{i}}{2}\right)^{k} P\left(\frac{2}{h_{i}}\left(x-x_{i+1 / 2}\right)\right)+O\left(h^{k+1}\right)
$$

on $\left[x_{i}, x_{i+1}\right]$ where $P(\xi)$ is defined in (2.5). Thus, the polynomial

$$
\tilde{v}(x)=v(x)+u^{(k)}\left(x_{i}\right)\left(\frac{h_{i}}{2}\right)^{k} P\left(\frac{2}{h_{i}}\left(x-x_{i+1 / 2}\right)\right)
$$

is an $O\left(h^{k+1}\right)$ approximation to $u(x)$ on $\left[x_{i}, x_{i+1}\right]$. Since $u(x) \in C^{(2 k+1)}[a, b]$, a 
straightforward argument using Markov's inequality (e.g., see the proof in [2, Lemma 4.1]) obtains $u^{(j)}(x)-\tilde{v}^{(j)}(x)=O\left(h^{k+1-j}\right)(0 \leqslant j \leqslant k)$.

Analysis of the low order factors from the $O\left(h^{k+1}\right)$ term in (2.7) produces the superconvergence results (2.6) at the breakpoints. In addition, we see from (2.4) that any real roots of $P^{(j)}(\xi)$ which fall in $(-1,1)$ correspond to points in $\left(x_{i}, x_{i+1}\right)$ at which the $j$ th derivative approximation is superconvergent. For example, a property of the polynomial $P(\xi)$ in $(2.5)$ is that $P(\xi)=(-1)^{k} P(-\xi)$, so that $P^{(k-1)}(0)=0$. Hence, $P^{(k-1)}(\xi)$ has a real root at $\xi=0$ which implies

$$
e^{(k-1)}\left(x_{i+1 / 2}\right)=O\left(h^{2}\right) \quad(1 \leqslant i \leqslant N),
$$

or

$$
v^{(k-1)}\left(x_{i+1 / 2}\right)-u^{(k-1)}\left(x_{i+1 / 2}\right)=O\left(h^{2}\right) .
$$

III. A Mesh Placement Algorithm. The mesh placement algorithm proposed in [3] and further discussed in [1], [4] is based on the error bound

$$
\|u-v\|_{i} \equiv \max _{x \in\left(x_{i}, x_{i+1}\right)}|u(x)-v(x)| \leqslant \text { const } h_{i}^{k}\left\|u^{(k)}\right\|_{i}+O\left(h^{k+1}\right)
$$

for the methods with $d>k$. The algorithm attempts to reduce the error by picking the mesh so that

$$
h_{i}^{k}\left\|u^{(k)}\right\|_{i}=\text { const } \quad(1 \leqslant i \leqslant N)
$$

From (2.4) we see that a mesh satisfying (3.2) equidistributes the leading terms in the error and minimizes $\max _{1 \leqslant i \leqslant N}\left(h_{i}^{k}\left\|u^{(k)}\right\|_{i}\right)$. For such a mesh

$$
\|u-v\|_{i}=\operatorname{const}(1+O(h)) \quad(1 \leqslant i \leqslant N) .
$$

In practice one does not use (3.2) but rather the condition

$$
\int_{x_{i}}^{x_{i+1}}\left|\tilde{u}^{(k)}(x)\right|^{1 / k} d x=\text { const } \quad(1 \leqslant i \leqslant N),
$$

where $\tilde{u}^{(k)}(x)$ is a piecewise constant approximation to $u^{(k)}(x)$. Condition (3.3) is asymptotically equivalent to (3.2) if $\tilde{u}^{(k)}(x)$ converges to $u^{(k)}(x)$.

De Boor suggests [1] the following approximation for $\tilde{u}^{(k)}(x)$ using the spline approximation $v(x)$ :

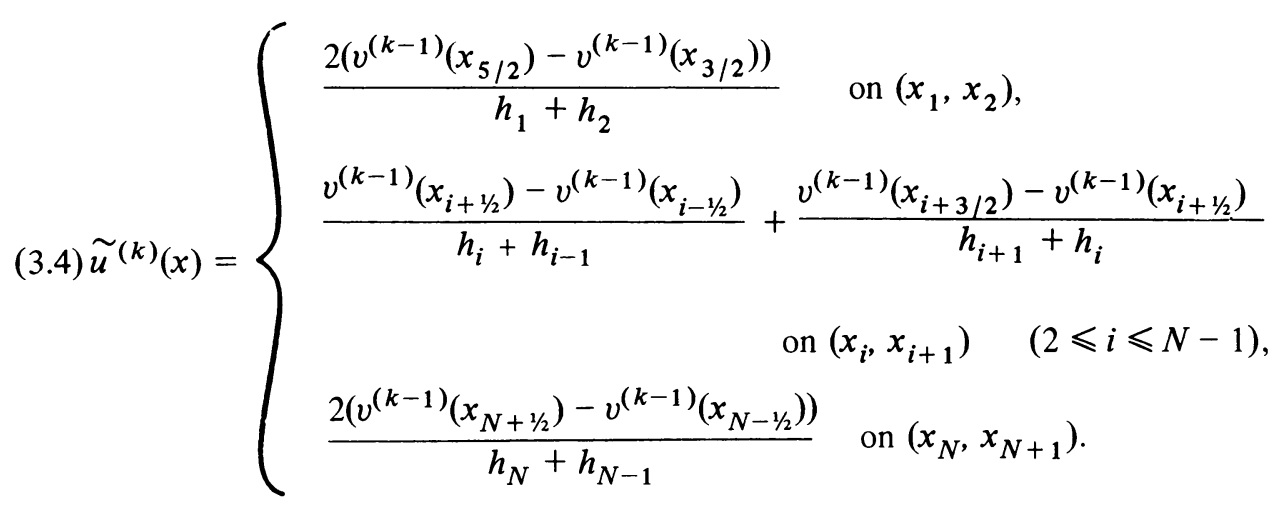


TABLE 1

\begin{tabular}{lllllllll}
\hline & \multicolumn{3}{c}{$N=6$} & \multicolumn{2}{c}{$N=12$} & \multicolumn{2}{c}{$N=24$} & \multicolumn{2}{c}{$N=48$} \\
\hline 1. & $.13(-6)$ & 7.5 & $.59(-9)$ & 7.8 & $.24(-11)$ & 8.0 & $.35(-13)^{*}$ & 6.1 \\
2. & $.28(-5)$ & 6.2 & $.29(-7)$ & 6.6 & $.27(-9)$ & 6.8 & $.22(-11)$ & 6.9 \\
3. & $.29(-4)$ & 4.8 & $.68(-6)$ & 5.4 & $.13(-7)$ & 5.7 & $.23(-9)$ & 5.8 \\
4. & $.36(-6)$ & 7.8 & $.15(-8)$ & 7.9 & $.59(-11)$ & 8.0 & $.29(-12)^{*}$ & 4.3 \\
5. & $.55(-4)$ & 4.9 & $.13(-5)$ & 5.4 & $.25(-7)$ & 5.7 & $.43(-9)$ & 5.8 \\
6. & $.15(-2)$ & 4.0 & $.66(-4)$ & 4.5 & $.25(-5)$ & 4.7 & $.86(-7)$ & 4.9 \\
7. & $.13(4)$ & .85 & $.50(3)$ & 1.4 & $.15(3)$ & 1.7 & $.42(2)$ & 1.9 \\
8. & $.55(5)$ & .51 & $.34(5)$ & .72 & $.19(5)$ & .85 & $.99(4)$ & .92 \\
9. & $.29(5)$ & -.17 & $.16(5)$ & .86 & $.60(4)$ & 1.4 & $.18(4)$ & 1.7 \\
$*$ & $*$
\end{tabular}

Substituting (2.9) into (3.4) shows that $\tilde{u}^{(k)}(x)=u^{(k)}(x)+O(h)$, so the mesh strategy of (3.3) (3.4) will asymptotically equidistribute the dominant terms in the error as $N \longrightarrow \infty$ if $K$ in (2.3) is sufficiently large. For cases where the scheme may fail, see [1]. The problem of simultaneously finding an approximation $v(x)$ to $u(x)$ and a mesh $\pi$ satisfying (3.2) is nonlinear [5] ; as such, convergence of the mesh selection scheme when one alternately approximates $u(x)$ and picks $\pi$ will depend upon the first approximation. Continuation has been useful for us in picking meshes for difficult problems.

IV. Numerical Example. Numerical results for collocation, including the adaptive scheme of Section III, have been dealt with in many other places so we confine ourselves to a single example to demonstrate the convergence properties derived in Section II.

The problem

$$
u^{\prime \prime}(x)+.1 u^{\prime}(x)+u(x)=100 e^{-10 x}, \quad 0 \leqslant x \leqslant 1, \quad u(0)=1, \quad u(1)=e^{-10},
$$

is solved using $C^{(1)}$ quintics and collocating at the four Gaussian points in each subinterval. For this method, from (2.5)

$$
P(\xi)=\frac{1}{720}\left(\xi^{2}-\frac{1}{7}\right)\left(\xi^{2}-1\right)^{2}
$$

so $P(\xi)$ has roots $\xi= \pm 1 / \sqrt{7}$ in $(-1,1)$ and $P^{\prime}(\xi)$ has roots $\xi= \pm \sqrt{3 / 7}$ in $(-1,1)$.

Therefore, from (2.6) and (2.7) we expect the following rates of convergence:

1. $O\left(h^{8}\right)$ for $e\left(x_{i}\right)(1 \leqslant i \leqslant N+1)$,

2. $O\left(h^{7}\right)$ for $e\left(x_{i+1 / 2} \pm h_{i} / 2 \sqrt{7}\right)(1 \leqslant i \leqslant N)$,

3. $O\left(h^{6}\right)$ uniformly for $e(x)$,

4. $O\left(h^{8}\right)$ for $e^{\prime}\left(x_{i}\right)(1 \leqslant i \leqslant N+1)$,

5. $O\left(h^{6}\right)$ for $e^{\prime}\left(x_{i+1 / 2} \pm\left(h_{i} / 2\right) \sqrt{3 / 7}\right)(1 \leqslant i \leqslant N)$,

6. $O\left(h^{5}\right)$ uniformly for $e^{\prime}(x)$,

7. $O\left(h^{2}\right)$ for $e^{(5)}\left(x_{i+1 / 2}\right)(1 \leqslant i \leqslant N)$, 
8. $O(h)$ uniformly for $e^{(5)}(x)$,

9. $O(h)$ for $u^{(6)}\left(x_{i}\right)-\tilde{u}^{(6)}\left(x_{i}\right)(1 \leqslant i \leqslant N+1)$.

Table 1 gives the actual errors (measured using the exact solution $u(x)=e^{-10 x}$ and its derivatives) and convergence rates from using equal spacing with $N=3$ initially and then halving the mesh four times. Uniform errors are estimated by monitoring the error at 20 points per subinterval.

Note that the convergence rate for $u^{(6)}\left(x_{i}\right)-\tilde{u}^{(6)}\left(x_{i}\right)$ appears to be $O\left(h^{2}\right)$ rather than the predicted $O(h)$. When using nonuniform meshes, as one would typically do in practice, the rate of convergence drops to the predicted $O(h)$, so that all quantities have the convergence rates expected. We omit these numerical results.

Department of Mathematics

Simon Fraser University

Burnaby, B. C., Canada V5A 1 S6

1. C. de BOOR, "Good approximation by splines with variable knots," II Conference on the Numerical Solution of Differential Equations, Lecture Notes in Math., vol. 363, Springer-Verlag, Berlin and New York, 1973.

2. C. de BOOR \& B. SWARTZ, "Collocation at Gaussian points," SIAM J. Numer. Anal., v. 10, 1973, pp. 582-606. MR 51 \#9528.

3. D. J. DODSON, Optimal Order Approximation by Spline Functions, Ph.D. Thesis, Purdue Univ., 1972.

4. R. D. RUSSELL \& J. CHRISTIANSEN, "Adaptive mesh selection strategies for solving boundary value problems," SIAM J. Numer. Anal. (To appear.)

5. A. B. WHITE, JR., On Selection of Equidistributing Meshes for Two-Point BoundaryValue Problems, Report 112, Univ. of Texas, Center Numer. Anal., 1976. 